\title{
Effect of vibration foam rolling on the range of motion in healthy adults: a systematic review and meta-analysis
}

\author{
Se-Ju Park, So-In Lee, Ho-Jin Jeong, Byeong-Geun Kim* \\ Department of Physical Therapy, Nambu University, Gwangju, Korea
}

Professionals use foam rollers to improve range of motion (ROM). Recently, a vibrating foam roller (VFR) that combines the vibration function with a foam roller (FR) has been used. The purpose of this systematic review and meta-analysis was to determine the effects of a VFR on the improvement of ROM in healthy individuals. A systematic literature search was carried out in five international databases: PubMed, Embase, PEDro, Cochrane Library, and Google Scholar. Eight clinical studies, composed of six randomized controlled trials and two randomized crossover trials that involved 230 healthy participants were selected for analysis. Methodological quality was identified using the PEDro scale. The mean scores, $4.75 \pm 0.71$, of the eight included studies, were classi-

\section{INTRODUCTION}

The range of motion (ROM) of a joint refers to the range within which the joint moves. Flexibility is the ability of a joint to move spontaneously with full ROM (Dantas et al., 2011). Limitation in the ROM or decreased flexibility may increase the incidence of injuries, muscle, and tendon tension (Gleim and McHugh, 1997), or the independence of people with neurological disorders (Harvey et al., 2003). It limits functional ability and decreases the quality of life (Stathokostas et al., 2012). To improve the ROM and flexibility, many people use the stretching method (Decoster et al., 2005; Harvey et al., 2002; Medeiros et al., 2016; Radford et al., 2006). However, stretching can cause problems in muscle performance (Behm et al., 2016; Simic et al., 2013). As an alternative to this problem, medical professionals use foam rollers (FRs) to reduce pain while improving the ROM (Cheatham, 2019). FRs are used as a form of massage that applies pressure to soft tissues using a person's own weight (Peacock et al., 2014; Pearcey et al., 2015). fied as fair. The results demonstrated that the VFR achieved better gains than the FR in improving ROM (standardized mean difference [SMD], $0.53 ; 95 \%$ confidence intervals [Cls], $0.29-0.77 ; I^{2}=55 \%$ ). The VFR was more effective in improving the ROM than the FR in the hip and knee joints (hip: SMD, 0.56; 95\% Cl, 0.28-0.85; $I^{2}=0 \%$; knee: SMD, 0.86; 95\% $\left.\mathrm{Cl}, 0.42-1.30 ; I^{2}=79 \%\right)$. The VFR may be an additional option to improve the ROM in healthy adults and athletes.

Keywords: Flexibility, Foam rolling, Range of motion, Vibration foam rolling

FRs have been reported to have advantages in muscle performance and recovery (Weerapong et al., 2005), thereby, FRs compensate for the disadvantages incurred with stretching.

Recently, vibration foam rollers (VFRs) that combine the vibration technology with FRs have been used. Vibration stimulation primarily contributes to muscle activity, strength, and neural mechanisms (Cochrane, 2011). However, despite the recent surge in research publications on VFRs, systematic evidence derived from them is still lacking. In previous systematic reviews including FRs and VFRs quantitative data synthesis was not performed, and the effect size could not be determined (Behm and wilke, 2019; Cheatham et al., 2015; Hendricks et al., 2020; Hughes and Ramer, 2019). In the meta-analysis, only one to three studies on VFRs were included and they were reported together with studies on FRs; hence, insufficient data was available to analyze the effects of VFRs (Skinner et al., 2020; Wiewelhove et al., 2019; Wilke et al., 2020). Therefore, the purpose of this study was to systematically review and evaluate the effects of VFRs on the improvement of
${ }^{*}$ Corresponding author: Byeong-Geun Kim (iD https://orcid.org/0000-0002-7358-7389 Department of Physical Therapy, Nambu University, 1 Nambudae-gil,

Gwangsan-gu, Gwangju 62271, Korea

Email: qudrms_92@naver.com

Received: May 31, 2021 / Accepted: June 22, 2021
This is an Open Access article distributed under the terms of the Creative Commons Attribution Non-Commercial License (https://creativecommons.org/licenses/by-nc/4.0/) which permits unrestricted non-commercial use, distribution, and reproduction in any medium, provided the original work is properly cited. 
the ROM in healthy individuals.

\section{MATERIALS AND METHODS}

\section{Protocol and registration}

This systematic review and meta-analysis was registered in the PROSPERO (no. CRD42021231413) database. We followed the Preferred Reporting Items for Systematic Review and Meta-Analysis (PRISMA) guidelines while reporting this study (Liberati et al., 2009).

\section{Eligibility criteria}

The strategy of this systematic review and meta-analysis was determined according to the PICO (participant, intervention, comparison, and outcome) strategy. In this review, participants $(\mathrm{P})$ were defined as healthy individuals. VFR was defined as the intervention (I) in the experimental group. The experimental group was compared $(\mathrm{C})$ with the FR group. Outcome $(\mathrm{O})$ was defined as the change in ROM and flexibility. Only studies involving randomized controlled trials (RCTs), randomized crossover trials and articles published in English were included. Studies were excluded if they were quasi-experimental studies (non-RCT, interrupted time series), observational studies (prospective and retrospective), case reports, reviews, or systematic literature reviews and qualitative studies, opinion pieces, editorials, comments, news, and letters.

\section{Search strategy}

In January 2021, two independent reviewers performed a systematic literature search. Articles relevant to the research question were identified using PubMed, Embase, PEDro, Cochrane Library, and Google Scholar. Each database was searched from the earliest available article until December 2020. As an example, the terms used for the PubMed search were: "Vibration Foam Rolling," "Vibration Foam Roller," "Vibration Rolling," "Vibration Roller," ("Vibration Foam Rolling" OR "Vibration Foam Roller") AND ("Range of Motion" OR "ROM"), ("Vibration Rolling" OR "Vibration Roller") AND ("Range of Motion" OR "ROM"), ("Vibration Foam Rolling" OR "Vibration Foam Roller") AND "Flexibility," ("Vibration Rolling" OR "Vibration Roller") AND "Flexibility." In Google Scholar, a hand-searching approach was used. We searched all the articles and extracted the appropriate publications.

\section{Study selection}

Two reviewers independently assessed the eligible studies by screening the titles and abstracts. Duplicates were identified and excluded using Endnote $\times 8$. These two reviewers checked the full-text when necessary. In cases of disagreements, a consensus was sought between the reviewers, or the coauthor reviewer was asked to resolve the issue.

\section{Data extraction and data synthesis}

Data extraction was performed by two reviewers independently using a standardized data collection form that included the year of publication; first author; sex, age, weight, and body mass index of the participants; study design; use of vibration technology; intervention protocols; outcomes; and results (pre-post mean changes, pre-post standard deviation (SD) changes). If reporting was incomplete (missing SDs of the changes from baseline), missing data were imputed using the formula $\mathrm{SD}_{\text {change }}=\sqrt{ }\left(\mathrm{SD}_{\text {baseline }}^{2}+\mathrm{SD}_{\text {postintervention }}^{2}\right)-$ $\left(2 \times\right.$ Corr $\left.\times \mathrm{SD}_{\text {baseline }} \times \mathrm{SD}_{\text {postintervention }}\right)$ (Higgins et al., 2021), where correlation $(\mathrm{Corr})=$ mean Corr of the included studies. In cases of disagreements, a consensus was sought between the reviewers, or the coauthor reviewer was asked to resolve the issue.

\section{Quality assessment}

Methodological quality was implemented by two reviewers independently, after assessing the items based on the PEDro scale. In cases of disagreements, the coauthor reviewer cast a decisive vote. The total score of the instrument is calculated using items $2-11$, capturing potential sources of bias. Item 1 refers to the external validity of the trial and thus is not included in the total PEDro score. The PEDro scale determines the scientific validity of physical therapy clinical trials (excellent, 9-10; good, 6-8; fair, 4-6; poor, <4).

\section{Statistical analyses}

The meta-analysis was performed using the R 4.1.1 (R Foundation for Statistical Computing, Vienna, Austria). In the metaanalyses, when comparing outcomes measured on different scales, the standardized mean difference (SMD) with $95 \%$ confidence intervals (CIs) was used. The results were considered statistically significant when $P<0.05$. Heterogeneity across studies was tested using the $I^{2}$ statistic, which is a quantitative measure of inconsistency across studies. Studies were considered to have low heterogeneity when the $I^{2}$ statistic was $25 \%-50 \%$, and those with an $I^{2}$ statistic $>75 \%$ were considered to have high heterogeneity. A subgroup analysis was conducted for: (a) hip vs. knee vs. ankle; and (b) according to the outcome. A sensitivity analysis was used to remove each study individually. 


\section{RESULTS}

\section{Search results}

A PRISMA flowchart of the study selection is shown in Fig. 1. A total of 93 records were identified with 14 records remaining after the duplicates were removed. Next, five records were excluded after screening the title and abstract, leaving nine articles for full-text review. Among the nine articles, one was excluded for the following reasons: the mean and SD could not be obtained $(n=1)$. Finally, eight records were included in our meta-analysis (Cheatham et al., 2019; De Benito et al., 2019; Han et al., 2017; Kim and Shin, 2020; Lee et al., 2018; Lim and Park, 2019; Lim et al., 2019; Romero-Moraleda et al., 2019).

\section{Characteristics of the studies}

The eight clinical studies, composed of six RCTs and two randomized crossover trials, involved 230 healthy participants aged 20 years or older. The number of participants in each study ranged from 15 to 45 . One study including 20 participants investigated VFRs with a vibration frequency of 3,700 rpm (Han et al., 2017). In the other seven studies, comprising 200 participants, the vibration frequencies varied from 18 to $33 \mathrm{~Hz}$ (Cheatham et al., 2019; De Benito et al., 2019; Kim and Shin, 2020; Lee et al., 2018; Lim and Park, 2019; Lim et al., 2019; Romero-Moraleda et al., 2019). The characteristics of the VFR protocols and the outcome measurements are presented in Table 1.

\section{Methodological quality}

The methodological quality of the eight included studies ranged from 4 to 6 out of 10 and their mean scores with a sum of $4.75 \pm$ 0.71 , were classified as fair (Table 2). All studies had similar baseline values between groups and reported information about randomization, results of inter-group statistical comparisons, and provided both point measures and measures of variability. In contrast, only a few studies indicated participant, therapist, or assessor blinding.

\section{VFR versus $F R$}

The total result demonstrated that VFRs achieved better gains than FRs in terms of improving the ROM (SMD, 0.53; 95\% CI, 0.29-0.77; $I^{2}=55 \%$ ), as shown in Fig. 2.

\section{Effect of VFR on the ROM in each joint}

The subgroup analysis was based on VFRs for the ROM in the hip, knee, and ankle joints (Fig. 3). The results for the knee indicated that VFRs improved the ROM (SMD, 0.59; 95\% CI, 0.24$\left.0.95 ; I^{2}=66 \%\right)$. In addition, significant differences were observed

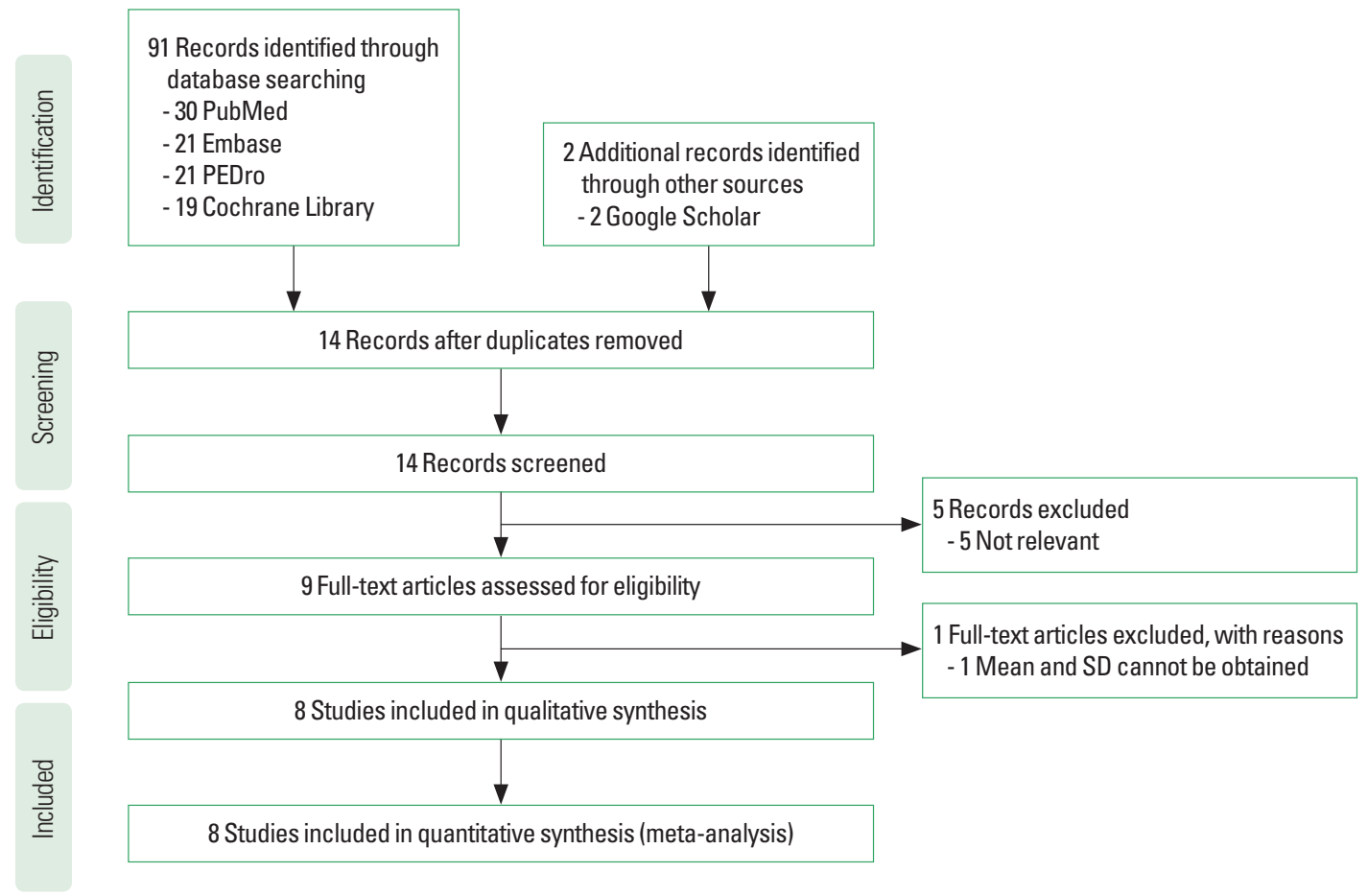

Fig. 1. Flowchart describing the study selection process. 
Park SJ, et al. • Effect of vibration rolling on range of motion

Table 1. Characteristics of the included studies

\begin{tabular}{|c|c|c|c|c|c|}
\hline Author & Design & Participants & VFR protocol & Vibration & Outcome \\
\hline $\begin{array}{l}\text { Cheatham et al., } \\
2019\end{array}$ & $\begin{array}{l}\text { RCT } \\
\text { 1. VFR }(n=15) \\
\text { 2. } F R(n=15) \\
\text { 3. } \operatorname{CON}(n=15)\end{array}$ & $\begin{array}{l}\mathrm{n}=45 \text { recreationally active adults } \\
\text { (male }=27 \text { and female }=18 \text { ) } \\
\text { 1. } 26.6 \pm 6.34 \mathrm{yr}, 24.8 \pm 3.6 \mathrm{~kg} / \mathrm{m}^{2} \\
\text { 2. } 24.53 \pm 3.96 \mathrm{yr}, 25.49 \pm 4.09 \mathrm{~kg} / \mathrm{m}^{2} \\
\text { 3. } 25.93 \pm 5.52 \mathrm{yr}, 25.79 \pm 6.82 \mathrm{~kg} / \mathrm{m}^{2}\end{array}$ & $\begin{array}{l}\text { VFR. } 1 \times 120 \text { s rolling quadriceps } \\
\text { FR. } 1 \times 120 \text { s rolling quadriceps }\end{array}$ & $33 \mathrm{~Hz}$ & Passive knee flexion ROM \\
\hline $\begin{array}{l}\text { De Benito et al., } \\
2019\end{array}$ & $\begin{array}{l}\text { Crossover } \\
\text { 1. VFR } \\
\text { 2. FR } \\
\text { 3. CON }\end{array}$ & $\begin{array}{l}\mathrm{n}=15 \text { healthy recreationally active } \\
\text { participants (male }=17 \text { and } \\
\text { female }=7) 21.78 \pm 2.41 \mathrm{yr} \text {, } \\
23.01 \pm 2.52 \mathrm{~kg} / \mathrm{m}^{2}\end{array}$ & $\begin{array}{l}\text { VFR. } 2 \times 60 \text { s rolling quadriceps } \\
\text { and hamstrings } \\
\text { FR. } 2 \times 60 \text { s rolling quadriceps and } \\
\text { hamstrings }\end{array}$ & $30 \mathrm{~Hz}$ & $\begin{array}{l}\text { Lunge ankle dorsiflexion ROM, sit } \\
\text { and reach test }\end{array}$ \\
\hline Han et al., 2017 & $\begin{array}{l}\text { RCT } \\
\text { 1. VFR }(n=15) \\
\text { 2. } F R(n=15)\end{array}$ & $\begin{array}{l}n=30 \text { students of university } \\
1.20 .50 \pm 1.09 \mathrm{yr}, 62.58 \pm 8.5 \mathrm{~kg} \\
\text { 2. } 20.6 \pm 0.83 \mathrm{yr}, 66.41 \pm 16.45 \mathrm{~kg}\end{array}$ & 4 wk, 3 times a week, $1 \times 20$ min & $3,700 \mathrm{RPM}$ & $\begin{array}{l}\text { Hip joint ROM (flexion, extension, } \\
\text { internal rotation, and external } \\
\text { rotation) }\end{array}$ \\
\hline Kim and Shin, 2020 & $\begin{array}{l}\text { RCT } \\
\text { 1. DVFR }(n=12) \\
\text { 2. } \operatorname{SVFR}(n=12) \\
\text { 3. } F R(n=12)\end{array}$ & $\begin{array}{l}n=36 \text { students of university } \\
\text { (male }=27 \text { and female }=9 \text { ) } \\
\text { 1. } 22.33 \pm 2.3 \mathrm{yr}, 67.83 \pm 12.53 \mathrm{~kg} \\
2.21 .5 \pm 1.44 \mathrm{yr}, 69.67 \pm 12.12 \mathrm{~kg} \\
3.21 .75 \pm 1.36 \mathrm{yr}, 69.5 \pm 12.15 \mathrm{~kg}\end{array}$ & $\begin{array}{l}\text { DVFR. } 3 \times 90 \text { s rolling hamstrings } \\
\text { SVFR. } 3 \times 90 \text { s not rolling ham- } \\
\text { strings } \\
\text { FR. } 3 \times 90 \text { s rolling hamstrings }\end{array}$ & $32 \mathrm{~Hz}$ & $\begin{array}{l}\text { Modified active knee extension, } \\
\text { stand to reach test }\end{array}$ \\
\hline Lee et al., 2018 & $\begin{array}{l}\text { Crossover } \\
\text { 1. VFR } \\
\text { 2. FR } \\
\text { 3. SS }\end{array}$ & $\begin{array}{l}\mathrm{n}=30 \text { male college students } \\
20.4 \pm 1.2 \mathrm{yr}, 68.8 \pm 8.9 \mathrm{~kg}\end{array}$ & $\begin{array}{l}\text { VFR. } 3 \times 30 \text { s rolling quadriceps } \\
\text { and hamstrings } \\
\text { FR. } 3 \times 30 \text { s rolling quadriceps and } \\
\text { hamstrings } \\
\text { SS. } 3 \times 30 \text { s static stretching } \\
\text { quadriceps and hamstrings }\end{array}$ & $28 \mathrm{~Hz}$ & Ely's test, popliteal angle test \\
\hline Lim and Park, 2019 & $\begin{array}{l}\text { RCT } \\
\text { 1. VFR }(n=10) \\
\text { 2. } F R(n=10)\end{array}$ & $\begin{array}{l}n=20 \text { college students (male }=14 \text { and } \\
\text { female }=6 \text { ) } \\
\text { 1. } 20.21 \pm 1.01 \mathrm{yr}, 63.67 \pm 11.1 \mathrm{~kg} \\
\text { 2. } 21.72 \pm 2.16 \mathrm{yr}, 68.93 \pm 17.16 \mathrm{~kg}\end{array}$ & $\begin{array}{l}\text { VFR. each } 5 \times 60 \text { s rolling both } \\
\text { hamstrings } \\
\text { FR. each } 5 \times 60 \text { s rolling both } \\
\text { hamstrings }\end{array}$ & $32 \mathrm{~Hz}$ & $\begin{array}{l}\text { Active straight leg raising, active } \\
\text { knee extension }\end{array}$ \\
\hline Lim et al., 2019 & $\begin{array}{l}\text { RCT } \\
\text { 1. VFR }(n=8) \\
\text { 2. } F R(n=8)\end{array}$ & $\begin{array}{l}\mathrm{n}=16 \text { recreationally active persons } \\
(\text { male }=11 \text { and female }=5) \\
\text { 1. } 20.37 \pm 1.06 \mathrm{yr}, 20.85 \pm 1.48 \mathrm{~kg} / \mathrm{m}^{2} \\
2.20 .75 \pm 1.39 \mathrm{yr}, 22.68 \pm 4.19 \mathrm{~kg} / \mathrm{m}^{2}\end{array}$ & $\begin{array}{l}\text { VFR. } 5 \times 60 \text { s rolling hamstrings } \\
\text { FR. } 5 \times 60 \text { s rolling hamstrings }\end{array}$ & $32 \mathrm{~Hz}$ & Sit and reach test \\
\hline $\begin{array}{l}\text { Romero-Moraleda } \\
\text { et al., } 2019\end{array}$ & $\begin{array}{l}\text { RCT } \\
\text { 1. VFR }(n=19) \\
\text { 2. } F R(n=19)\end{array}$ & $\begin{array}{l}\mathrm{n}=38 \text { healthy individuals (male }=32 \\
\quad \text { and female }=6 \text { ) } \\
1.21 .9 \pm 3.7 \mathrm{yr}, 75.26 \pm 8 \mathrm{~kg} \\
\text { 2. } 22.2 \pm 3.2 \mathrm{yr}, 69.7 \pm 11.4 \mathrm{~kg}\end{array}$ & $\begin{array}{l}\text { VFR. } 5 \times 60 \text { s rolling quadriceps } \\
\text { FR. } 5 \times 60 \text { s rolling quadriceps }\end{array}$ & $18 \mathrm{~Hz}$ & $\begin{array}{l}\text { Passive and active hip extension } \\
\text { and knee flexion ROM }\end{array}$ \\
\hline
\end{tabular}

VFR, vibrating foam roller; RCT, randomized controlled trials; FR, foam roller; CON, control; ROM, range of motion; DVFR, dynamic vibration foam roller; SVFR, static vibration foam roller; SS, static stretching.

Table 2. Assessments of methodological quality (PEDro scale)

\begin{tabular}{|c|c|c|c|c|c|c|c|c|c|c|c|c|}
\hline Study & 1 & 2 & 3 & 4 & 5 & 6 & 7 & 8 & 9 & 10 & 11 & Total score \\
\hline Cheatham et al., 2019 & $\mathrm{~N}$ & Y & $\mathrm{N}$ & Y & Y & N & N & $\mathrm{N}$ & Y & Y & Y & 6 \\
\hline De Benito et al., 2019 & $\mathrm{~N}$ & Y & $\mathrm{N}$ & Y & $\mathrm{N}$ & N & N & $\mathrm{N}$ & $\mathrm{N}$ & Y & Y & 4 \\
\hline Han et al., 2017 & Y & Y & $\mathrm{N}$ & Y & $\mathrm{N}$ & N & N & Y & $\mathrm{N}$ & Y & Y & 5 \\
\hline Kim and Shin, 2020 & Y & Y & $\mathrm{N}$ & Y & N & N & N & Y & $\mathrm{N}$ & Y & Y & 5 \\
\hline Lee et al., 2018 & $\mathrm{~N}$ & Y & $\mathrm{N}$ & Y & N & N & N & $\mathrm{N}$ & $\mathrm{N}$ & Y & Y & 4 \\
\hline Lim and Park, 2019 & Y & Y & $\mathrm{N}$ & Y & N & N & N & $\mathrm{N}$ & $\mathrm{N}$ & Y & Y & 4 \\
\hline Lim et al., 2019 & $\mathrm{~N}$ & Y & $\mathrm{N}$ & Y & $\mathrm{N}$ & N & Y & $\mathrm{N}$ & $\mathrm{N}$ & Y & Y & 5 \\
\hline Romero-Moraleda et al., 2019 & $\mathrm{~N}$ & Y & $\mathrm{N}$ & Y & $\mathrm{N}$ & $\mathrm{N}$ & Y & $\mathrm{N}$ & $\mathrm{N}$ & Y & Y & 5 \\
\hline
\end{tabular}

PEDro scale: 1, eligibility criteria were specified (this item is not used to calculate the PEDro score.); 2 , participants were randomly allocated to groups; 3 , allocation was concealed; 4 , the groups were similar at baseline regarding the most important prognostic indicators; 5 , there was blinding of all participants; 6 , there was blinding of all therapists who administered the therapy; 7, there was blinding of all assessors who measured at least one key outcome; 8, measures of at least one key outcome were obtained from more than $85 \%$ of the participants initially allocated to groups; 9 , all participants for whom outcome measures were available received the treatment or control condition as allocated or, where this was not the case, data for at least one key outcome was analyzed by "intention to treat;" 10, the results of inter-group statistical comparisons are reported for at least one key outcome; 11, the study provides both point measures and measures of variability for at least one key outcome. 


\section{Study}

Cheatham, 2019/Ely test, ${ }^{\circ}$

De Benito, 2019/Lunge, ...

De Benito, 2019/Sit \& R, ...

Han, 2017/HER, ${ }^{\circ}$

Han, 2017/HIR, ${ }^{\circ}$

Han, 2017/HEX, 。

Han, 2017/HFL, 。

Kim, 2020/MAKE, 。

Kim, 2020/Stand \& R, ...

Lee, 2018/Ely test, ${ }^{\circ}$

Lee, 2018/MAKE, 。

Lim, 2019(a)/ASLR(L),

Lim, 2019(a)/ASLR(R), 。

Lim, 2019(a)/AKE(L), 。

Lim, 2019(a)/AKE(R), ${ }^{\circ}$

Lim, 2019(b)/Sit \& R, ...

Romero-moraleda, 2019/AHE, 。

Romero-moraleda, 2019/PHE, 。

Romero-moraleda, 2019/AKF, 。

Romero-moraleda, 2019/PKF, 。

Random effects model
Heterogeneity: $I^{2}=55 \%, \tau^{2}=0.1632, p<0.01$

$\begin{array}{lr}\text { Experimental } & \text { Control } \\ \text { Total Mean SD Total Mean SD }\end{array}$

$\begin{array}{llllll}15 & 7.44 & 0.53 & 15 & 5.21 & 1.13\end{array}$

$\begin{array}{llllll}24 & 0.21 & 0.96 & 24 & 0.38 & 1.39\end{array}$

$\begin{array}{llllll}24 & 1.16 & 3.58 & 24 & 1.21 & 4.98\end{array}$

$\begin{array}{llllll}15 & 3.80 & 1.78 & 15 & 1.40 & 3.26\end{array}$

$\begin{array}{llllll}15 & 10.80 & 5.28 & 15 & 6.80 & 3.08\end{array}$

$\begin{array}{llllll}15 & 8.30 & 3.67 & 15 & 6.70 & 2.56\end{array}$

$\begin{array}{llllll}15 & 16.80 & 5.73 & 15 & 14.70 & 7.13\end{array}$

$\begin{array}{llllll}12 & 9.25 & 2.01 & 12 & 4.83 & 3.44\end{array}$

$\begin{array}{llllll}12 & 6.21 & 0.91 & 12 & 3.67 & 2.18\end{array}$

$\begin{array}{llllll}30 & 3.08 & 3.15 & 30 & 0.75 & 4.93\end{array}$

$\begin{array}{llllll}30 & 7.66 & 7.02 & 30 & 6.25 & 8.32\end{array}$

$\begin{array}{llllll}10 & 6.90 & 4.19 & 10 & 1.90 & 7.82\end{array}$

$\begin{array}{llllll}10 & 10.00 & 5.31 & 10 & 7.30 & 10.55\end{array}$

$\begin{array}{llllll}10 & 5.00 & 4.26 & 10 & 3.00 & 6.22\end{array}$

$\begin{array}{llllll}10 & 5.15 & 4.53 & 10 & 6.30 & 10.20\end{array}$

$\begin{array}{llllll}8 & 2.29 & 2.19 & 8 & 3.50 & 2.96\end{array}$

$\begin{array}{llllll}19 & 5.00 & 2.24 & 19 & 3.84 & 2.86\end{array}$

$\begin{array}{llllll}19 & 4.37 & 2.45 & 19 & 3.10 & 2.94\end{array}$

$\begin{array}{llllll}19 & 3.46 & 2.56 & 19 & 1.78 & 1.86\end{array}$

$\begin{array}{llllll}19 & 2.26 & 2.58 & 19 & 1.11 & 2.28\end{array}$

331

331
Standardised Mean Difference

SMD

95\%-Cl Weight

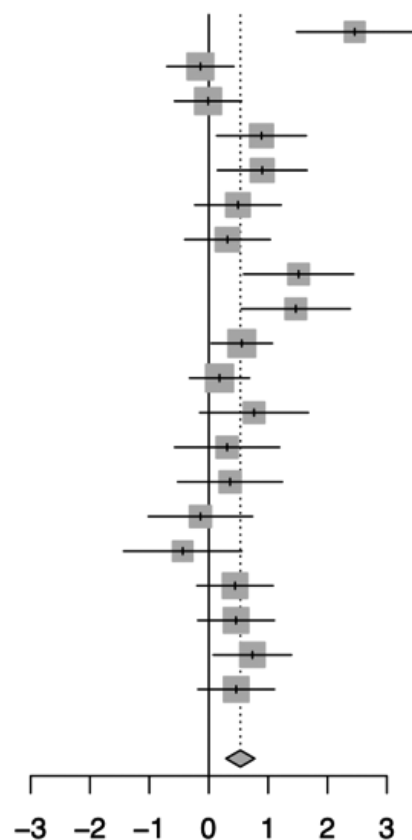

$2.46[1.48 ; 3.44] \quad 3.7 \%$

$-0.14[-0.71 ; 0.43] \quad 6.2 \%$

$-0.01[-0.58 ; 0.55] \quad 6.2 \%$

$0.89[0.13 ; 1.64] \quad 4.9 \%$

$0.90[0.14 ; 1.66] \quad 4.9 \%$

$0.49[-0.24 ; 1.22] \quad 5.1 \%$

$0.32[-0.41 ; 1.04] \quad 5.1 \%$

$1.51[0.59 ; 2.44] \quad 4.0 \%$

$1.47[0.55 ; 2.39] \quad 4.0 \%$

0.56 [ $0.04 ; 1.07] \quad 6.6 \%$

$0.18[-0.33 ; 0.69] \quad 6.7 \%$

$0.76[-0.15 ; 1.68] \quad 4.0 \%$

$0.31[-0.57 ; 1.19] \quad 4.2 \%$

$0.36[-0.53 ; 1.24] \quad 4.2 \%$

$-0.14[-1.02 ; 0.74] \quad 4.2 \%$

$-0.44[-1.43 ; 0.56] \quad 3.6 \%$

$0.44[-0.20 ; 1.09] \quad 5.6 \%$

$0.46[-0.19 ; 1.11] \quad 5.6 \%$

$0.74[0.08 ; 1.39] \quad 5.5 \%$

$0.46[-0.18 ; 1.11] \quad 5.6 \%$

$0.53[0.29 ; 0.77] 100.0 \%$

Fig. 2. Effects of vibrating foam roller (experimental) vs. foam roller (control) on range of motion. Forest plots with pooled standardized mean differences (SMDs), 95\% confidence intervals (Cls) are displayed. SD, standard deviation.

Subgroup

Knee

Random effects model

Heterogeneity: $I^{2}=66 \%, \tau^{2}=0.2719, p<0.01$

Ankle

Random effects model

Heterogeneity: not applicable

Hip

Random effects model

Heterogeneity: $I^{2}=0 \%, \tau^{2}=0, p=0.82$

Random effects model

Heterogeneity: $I^{2}=55 \%, \tau^{2}=0.1632, p<0.01$
Standardised Mean Difference

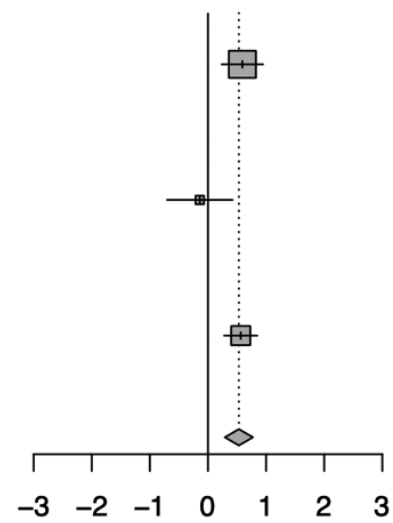

SMD $\quad 95 \%-C l$

$0.59[0.24 ; 0.95]$

$-0.14[-0.71 ; 0.43]$

$0.56[0.28 ; 0.85]$

$0.53[0.29 ; 0.77]$

Fig. 3. Effect of vibrating foam roller for the range of motion in each joint. Forest plots with pooled standardized mean differences (SMDs), 95\% confidence intervals (Cls) are displayed.

due to VFRs for the hip ROM (SMD, 0.56; 95\% CI, 0.28-0.85; $I^{2}=0 \%$ ). Furthermore, no significant differences were observed in VFRs for the ROM in the ankle (SMD, $-0.14 ; 95 \% \mathrm{CI},-0.71$ to $0.43)$. Heterogeneity was observed in the knee joints. There was no heterogeneity in the hip and ankle joints, or could not be confirmed.

\section{Effect of VFR on the ROM in each outcome of the knee joint}

To confirm the heterogeneity of the knee joint, a subgroup analysis was performed for each knee joint outcome (Fig. 4). Significant differences were observed in the stand \& reach test and active knee flexion (AKF) (stand and reach test: SMD, 1.47; $95 \%$ CI, 0.55-2.39; AKF: SMD, 0.74; 95\% CI, 0.08-1.39). No sig- 


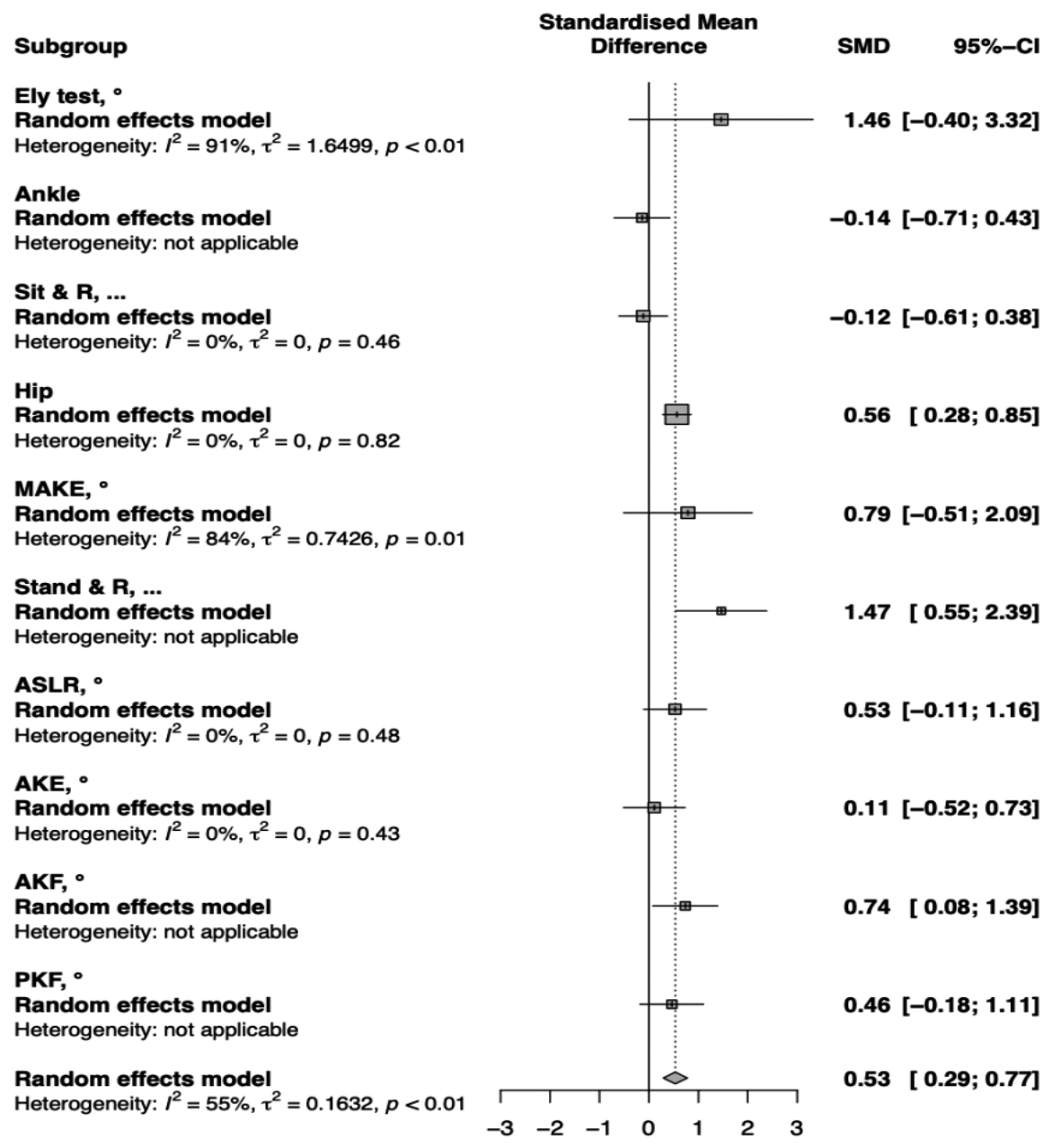

Fig. 4. Effect of vibrating foam roller on range of motion of each outcome knee joint outcome. Forest plots with pooled standardized mean differences (SMDs), 95\% confidence intervals (Cls) are displayed. Sit \& R, sit \& reach test; MAKE, modified active knee extension; Stand \& R, stand \& reach test; ASLR, active straight leg raising; AKE, active knee extension; AKF, active knee flexion; PKF, passive knee flexion.

nificant differences were observed in the other outcomes. It was found that there was high heterogeneity in the Ely test and modified active knee extension (MAKE) (Ely test, $I^{2}=91 \%$; MAKE, $\left.I^{2}=84 \%\right)$. In the other outcomes, there was no heterogeneity or could not be confirmed.

\section{Sensitivity analysis}

When the results reported by one study (Cheatham et al., 2019) were removed from the study, the heterogeneity of $55 \%$ was lowest at 32\% (SMD, 0.44; 95\% CI, 0.25-0.64; $I^{2}=32 \%$ ).

\section{DISCUSSION}

The use of VFRs has increased in recent years. However, direct evidence of its efficacy is still lacking. The results of this study provide practical evidence for the effects of VFRs on the ROM in healthy adults. Our results showed that VFRs can induce greater improvement in the ROM compared to FRs. In a systematic review of FRs, a meta- analysis reported that there was no difference between the of use of VFRs and FRs (Wilke et al., 2020). These results differ from the results of the present study. There was no significant difference in previous reviews (VFR vs. FR) due to the smaller number of included studies (three studies and four results) (Wilke et al., 2020). This study included more studies than previous studies, which we believe is the cause for the contrasting results (eight studies, 20 results).

Previous studies have reported only the overall effect without joint position-based (Wilke et al., 2020). In this study, subgroup analysis was performed based on joint position. It was effective in hip and knee joints, but not in ankle joints. The reason for this is 
that the number of studies included in the ankle joint is small, and the study evaluating the ankle joint did not intervene VFR to the ankle but to the quadriceps and hamstrings (De Benito et al., 2019), so I think that the effect on the ankle joint did not appear. A study evaluating and intervention on the ankle joint is needed.

The VFR is a tool that combines vibrations with FR. The effects of FR and vibration are not known. There are several arguments regarding the mechanism by which FR improves the ROM (Behm and Wilke, 2019). Among the studies included in this analysis, a study claimed that vibration is more effective in improving blood flow (De Benito et al., 2019; Lee et al., 2018; Romero-Moraleda et al., 2019). Most of the referenced studies had included results that were based on whole-body vibration and not local vibration. Other previous studies compared the effects of VFRs and FRs in improving blood flow to the skin and reported that although they were more effective, there was no statistically significant difference (Lai et al., 2020). We consider that the pressure of the FR along with vibration worked synergistically to improve the blood flow. Another hypothesis was that vibration activates the muscle spindle and causes tonic vibration reflex (Cheatham et al., 2019; Lim and Park, 2019; Lim et al., 2019). This hypothesis was put forth based on the results of measurement after an intervention (vibration) but the results were not confirmed with the addition of vibration (Pamukoff et al., 2014). However, the mechanism of action for vibration is not clear. Therefore, further research is required.

The results of this study suggest that VFR was effective in improving the ROM but showed high heterogeneity. Although an additional heterogeneity analysis was not conducted, there was high heterogeneity in the outcome of a specific evaluation method. Regarding the methodological quality of this study, most of the studies were not completely blinded to the participants, therapists, and assessors. These results were considered to have influenced the increase in heterogeneity. Therefore, studies with better methodologies should be conducted in the future. There was a limitation of this study. The number of studies included was small, thus an additional heterogeneity analysis was not performed, and publication bias was not confirmed.

\section{CONCLUSIONS}

VFRs are more effective in improving the ROM than general FRs. These results indicate that VFRs may be an additional option for normal adults and athletes to improve their ROM. Further research must be conducted in the future to study their efficacy.

\section{CONFLICT OF INTEREST}

No potential conflict of interest to this article was reported.

\section{ACKNOWLEDGMENTS}

The authors received no financial support for this article.

\section{REFERENCES}

Behm DG, Blazevich AJ, Kay AD, McHugh M. Acute effects of muscle stretching on physical performance, range of motion, and injury incidence in healthy active individuals: a systematic review. Appl Physiol Nutr Metab 2016;41:1-11.

Behm DG, Wilke J. Do self-myofascial release devices release myofascia? Rolling mechanisms: a narrative review. Sports Med 2019;49:1173-1181.

Cheatham SW. Roller massage: a descriptive survey of Allied Health professionals. J Sport Rehabil 2019;28:640-649.

Cheatham SW, Kolber MJ, Cain M, Lee M. The effects of self-myofascial release using a foam roll or roller massager on joint range of motion, muscle recovery, and performance: a systematic review. Int J Sports Phys Ther 2015;10:827-838.

Cheatham SW, Stull KR, Kolber MJ. Comparison of a vibration roller and a nonvibration roller intervention on knee range of motion and pressure pain threshold: a randomized controlled trial. J Sport Rehabil 2019;28:39-45.

Cochrane DJ. The potential neural mechanisms of acute indirect vibration. J Sports Sci Med 2011;10:19-30.

Dantas E, Daoud R, Trott, A, Nodari R, Conceição M. Flexibility: components, proprioceptive mechanisms and methods. Biomed Hum Kinet 2011;3:39-43.

De Benito AM, Valldecabres R, Ceca D, Richards J, Barrachina Igual JB, Pablos A. Effect of vibration vs non-vibration foam rolling techniques on flexibility, dynamic balance and perceived joint stability after fatigue. Peer] 2019;7:e8000.

Decoster LC, Cleland J, Altieri C, Russell P. The effects of hamstring stretching on range of motion: a systematic literature review. J Orthop Sports Phys Ther 2005;35:377-387.

Gleim GW, McHugh MP. Flexibility and its effects on sports injury and performance. Sports Med 1997;24:289-299.

Han SW, Lee YS, Lee DJ. The influence of the vibration form roller exercise on the pains in the muscles around the hip joint and the joint performance. J Phys Ther Sci 2017;29:1844-1847.

Harvey L, Herbert R, Crosbie J. Does stretching induce lasting increases in joint ROM? A systematic review. Physiother Res Int 2002;7:1-13. 
Harvey LA, Byak AJ, Ostrovskaya M, Glinsky J, Katte, L, Herbert RD. Randomised trial of the effects of four weeks of daily stretch on extensibility of hamstring muscles in people with spinal cord injuries. Aust J Physiother 2003;49:176-181.

Hendricks S, Hill H, Hollander SD, Lombard W, Parker R. Effects of foam rolling on performance and recovery: a systematic review of the literature to guide practitioners on the use of foam rolling. J Bodyw Mov Ther 2020;24:151-174.

Higgins JPT, Thomas J, Chandler J, Cumpston M, Li T, Page MJ, Welch VA. Cochrane Handbook for Systematic Reviews of Interventions version 6.2 [Internet]. London: Cochrane; [cited 2021 Jun 20]. Available from: https://www.training.cochrane.org/handbook.

Hughes GA, Ramer LM. Duration of myofascial rolling for optimal recovery, range of motion, and performance: a systematic review of the literature. Int J Sports Phys Ther 2019;14:845-859.

Kim H, Shin W. Immediate effects of myofascial release using vibration foam rolling methods on hamstrings range of motion, flexibility, pressure pain thresholds and dynamic balance. J Int Acad Phys Ther Res 2020;11:2042-2051.

Lai YH, Wang AY, Yang CC, Guo LY. The recovery benefit on skin blood flow using vibrating foam rollers for postexercise muscle fatigue in runners. Int J Environ Res Public Health 2020;17:9118.

Lee CL, Chu IH, Lyu BJ, Chang WD, Chang, NJ. Comparison of vibration rolling, nonvibration rolling, and static stretching as a warm-up exercise on flexibility, joint proprioception, muscle strength, and balance in young adults. J Sports Sci 2018;36:2575-2582.

Liberati A, Altman DG, Tetzlaff J, Mulrow C, Gøtzsche PC, Ioannidis JP, Clarke M, Devereaux PJ, Kleijnen J, Moher D. The PRISMA statement for reporting systematic reviews and meta-analyses of studies that evaluate health care interventions: explanation and elaboration. J Clin Epidemiol 2009;62:e1-e34.

Lim JH, Park CB. The immediate effects of foam roller with vibration on hamstring flexibility and jump performance in healthy adults. J Exerc Rehabil 2019;15:50-54.

Lim JH, Park CB, Kim BG. The Effects of vibration foam roller applied to hamstring on the quadriceps electromyography activity and hamstring flexibility. J Exerc Rehabil 2019;15:560-565.
Medeiros DM, Cini A, Sbruzzi G, Lima CS. Influence of static stretching on hamstring flexibility in healthy young adults: systematic review and meta-analysis. Physiother Theor Pract 2016;32:438-445.

Pamukoff DN, Ryan ED, Blackburn JT. The acute effects of local muscle vibration frequency on peak torque, rate of torque development, and EMG activity. J Electromyogr Kinesiol 2014;24:888-894.

Peacock CA, Krein DD, Silver TA, Sanders GJ, Von Carlowitz KA. An acute bout of self-myofascial release in the form of foam rolling improves performance testing. Int J Exerc Sci 2014;7:202-211.

Pearcey GE, Bradbury-Squires DJ, Kawamoto JE, Drinkwater EJ, Behm DG, Button DC. Foam rolling for delayed-onset muscle soreness and recovery of dynamic performance measures. J Athl Train 2015;50:5-13.

Radford JA, Burns J, Buchbinder R, Landorf KB, Cook C. Does stretching increase ankle dorsiflexion range of motion? A systematic review. Br J Sports Med 2006;40:870-875.

Romero-Moraleda B, González-García J, Cuéllar-Rayo Á, BalsalobreFernández C, Muñoz-García D, Morencos E. Effects of vibration and non-vibration foam rolling on recovery after exercise with induced muscle damage. J Sports Sci Med 2019;18:172-180.

Simic L, Sarabon N, Markovic G. Does pre-exercise static stretching inhibit maximal muscular performance? A meta-analytical review. Scand J Med Sci Sports 2013;23:131-148.

Skinner B, Moss R, Hammond L. A systematic review and meta-analysis of the effects of foam rolling on range of motion, recovery and markers of athletic performance. J Bodyw Mov Ther 2020;24:105-122.

Stathokostas L, Little RM, Vandervoort AA, Paterson DH. Flexibility training and functional ability in older adults: a systematic review. J Aging Res 2012;2012:306818.

Weerapong P, Hume PA, Kolt GS. The mechanisms of massage and effects on performance, muscle recovery and injury prevention. Sports Med 2005;35:235-256.

Wiewelhove T, Döweling A, Schneider C, Hottenrott L, Meyer T, Kellmann M, Pfeiffer M, Ferrauti A. A meta-analysis of the effects of foam rolling on performance and recovery. Front Physiol 2019;10:376.

Wilke J, Müller AL, Giesche F, Power G, Ahmedi H, Behm DG. Acute effects of foam rolling on range of motion in healthy adults: a systematic review with multilevel meta-analysis. Sports Med 2020;50:387-402. 